Title : will be set by the publisher

Editors : will be set by the publisher

EAS Publications Series, Vol. ?, 2018

\title{
ON THE CEPHEID METALLICITY DICHOTOMY
}

\author{
Géza Kovács ${ }^{1}$
}

\begin{abstract}
With the aid of stellar atmosphere models, we derive iron abundances $[\mathrm{Fe} / \mathrm{H}]$ from the OGLE $B, V, I$ photometry on the Magellanic Cloud Cepheids. We show that in both clouds the average metallicities of the first overtone variables are lower than those of the fundamental ones (by $\sim 0.2$ and $\sim 0.3 \mathrm{dex}$ in the LMC and SMC, respectively). Consequently, there is a correlation between the overall $[\mathrm{Fe} / \mathrm{H}]$ and luminosity; the lower luminosity stars tend to be also of lower metallicity. These metallicity dependencies are concordant with the ones derived for the two types of double-mode Cepheids from pulsation theory. Yet another support of this dichotomy comes from the evolution theories that require lower metallicities for blue-looping lowluminosity stars than for high-luminosity ones. We also comment on the possibility of using period-luminosity-color relations to derive more accurate metallicities.
\end{abstract}

\section{Introduction}

It is well known that chemical composition plays a very important role in stellar evolution and in various methods of stellar parameter computations, most remarkably in asteroseismological investigations. From the point of view of chemical composition, stellar clusters and certain populations of galaxies have been treated quite often as chemically homogeneous ensembles. However, accumulating abundance measurements show that there are chemical inhomogeneities even in globular clusters (e.g., Yong \& Grundahl 2008), where the uniform composition thought to be well-justified on the basis of common origin of the constituting objects. It has also been evident during the recent years - mostly due to the systematic spectroscopic surveys of Andrievsky and coworkers - that there is a considerable metallicity spread among the Galactic Cepheids and that the observed metallicity correlates with the distance from the Galactic center (Luck, Kovtyukh, \& Andrievsky 2006). The importance of the heavy element abundance in stellar pulsation has become

\footnotetext{
${ }^{1}$ Konkoly Observatory, Budapest, Hungary
} 
Title : will be set by the publisher

especially clear after 1992, with the verification of Simon's hypothesis on the iron opacity bump (Simon 1982, Rogers \& Iglesias 1992). Perhaps the largest impact made by the opacity increase was the near elimination of the so-called 'Cepheid mass discrepancy' (Moskalik, Buchler \& Marom 1992) and the excitation of the $\beta$ Cephei stars (Moskalik \& Dziembowski 1992). In addition, based on these revised opacities, there are theoretical results which show that the consideration of metallicity spread is a must in various groups of Cepheids. Currently it has been shown that the observed periods of the first/second overtone double-mode Cepheids in the Magellanic Clouds can be fitted only if we assume that the metallicities of these low-luminosity stars are lower than their fundamental/first overtone counterparts (Kovács 2006). This observation supports the earlier result of Cordier, Goupil \& Lebreton (2003), suggesting that Cepheids cannot 'blue-loop' at low-luminosities in the SMC, unless they have systematically lower metallicities than the higher luminosity ones. Stimulated by the above results, the goal of the present study is to use the photometric metallicities in the investigation of the metallicity spread among the Magellanic Cloud Cepheids.

\section{Metallicities from BVI colors}

Our method has already been described in Dékány et al. (2008) and tested on a sample of 21 fundamental mode Galactic field RR Lyrae stars. Here we summarize the main steps of the method and present tests concerning the limits of applicability of the photometrically derived abundances.

Assuming that the poorly known quantities, such as the turbulent velocity and convective mixing length are fixed to some generally acceptable value and the relative abundances are also given (e.g., scaled to the Sun), then stellar atmosphere models and basic pulsational relations yield the following set of equations:

$$
B-V=F\left(T_{\text {eff }}, g,[\mathrm{Fe} / \mathrm{H}]\right), \quad V-I=G\left(T_{\text {eff }}, g,[\mathrm{Fe} / \mathrm{H}]\right), \quad g=H(P),
$$

where the last equation is applicable in a wide range of parameters in the region of Cepheids (see Kovács 2000). The functions $F$ and $G$ denote the dependence of the theoretical color indices on the physical parameters as given by the models of Castelli, Gratton \& Kurucz (1997). All models have solar-type heavy element distribution, microturbulent velocity of $2 \mathrm{~km} \mathrm{~s}^{-1}$ and no convective overshooting. Quadratic interpolation among these models proved to be accurate enough to find the solution of set of Eqs. (2.1), which is searched for by minimizing the following quantity:

$$
\mathcal{D}^{2}=\left[\Delta \log T_{\mathrm{eff}}\right]^{2}+\left[w_{\mathrm{B}-\mathrm{V}} \Delta(B-V)\right]^{2}+\left[w_{\mathrm{V}-\mathrm{I}} \Delta(V-I)\right]^{2},
$$

where $\Delta \log T_{\text {eff }}$ is the difference between the $\log T_{\text {eff }}$ values computed from the $B-V$ and $V-I$ colors, whereas $\Delta(B-V)$ and $\Delta(V-I)$ denote the differences between the observed values and the ones given by the stellar atmosphere model. 
The weights $w_{\mathrm{B}-\mathrm{V}}$ and $w_{\mathrm{V}-\mathrm{I}}$ are set equal to 0.33 and 0.25 , respectively, following the proportionality of $\log T_{\text {eff }}$ in the approximate linear expressions of Kovács \& Walker (1999). The last two terms in $\mathcal{D}$ increases the stability of the method against observational noise. In general, we accept solutions only with $\mathcal{D}<0.001$.
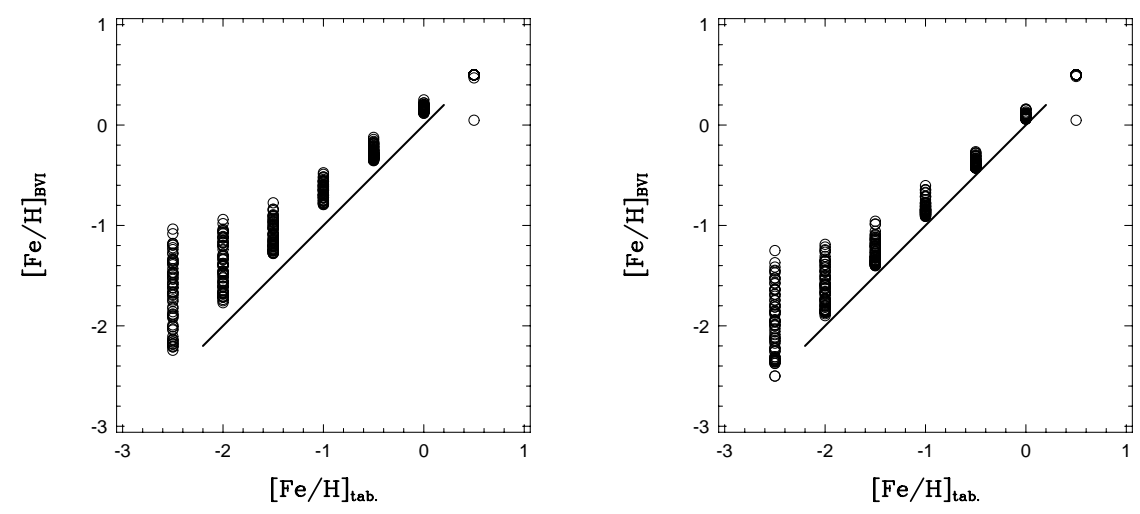

Fig. 1. Left: theoretical vs. computed photometric metallicities by shifting the theoretical $V$ magnitudes by $\Delta V=-0.01 \mathrm{mag}$ and use this (with the other input parameters left at their theoretical values) in the computation of $[\mathrm{Fe} / \mathrm{H}]_{\mathrm{BVI}}$. Right: the same plot for the $B$ color with $\Delta B=+0.01$ mag.

For variable stars it is a question of what kind of average magnitudes should we use if we need a good approximation of the static colors. We use the intensity averaged magnitudes mostly because of the easy availability of these values from the OGLE database. Apparently the intensity averages yield closer agreement with the static colors, at least for RR Lyrae stars, concerning B, V and K colors (Bono, Caputo \& Stellingwerf 1995).

The method was tested on various datasets. For a sample of 42 Pop. I dwarfs with spectroscopically determined $[\mathrm{Fe} / \mathrm{H}], \log g$ and $T_{\text {eff }}$ values and $B V I$ photometry (e.g., Clementini et al. 1995, Taylor 2003), we got $\sigma=0.14$ dex between the spectroscopic and our photometric $[\mathrm{Fe} / \mathrm{H}]$. A much more limited sample of 10 Galactic Cepheids yielded $\sigma=0.19$ dex. On the set of the 21 Galactic RR Lyrae stars mentioned above, we derived $\sigma=0.15$ dex. No apparent systematic differences were observed between the spectroscopic and photometric abundances. We conclude that the photometric abundances derived from the $B V I$ colors are reasonably accurate on various types of stars. We will see that this assessment is confirmed also by the average metallicities derived for the MC Cepheids.

To obtain additional information on the sensitivity of the photometric abundances, here we examine the differences obtained between the tabulated theoretical values and the ones obtained by various perturbations of the corresponding input parameters (as given by the stellar atmosphere models).

In Fig. 1 we show two examples on the effect of changing the photometric zero points. We see a dependence on the metallicity both in the overall difference and 
Title : will be set by the publisher

Table 1. Systematic and random effects for the photometric $[\mathrm{Fe} / \mathrm{H}]$ and $T_{\text {eff }}$

\begin{tabular}{lccc}
\hline \hline Systematic errors: & & & \\
Parameter & shift & $\Delta[\mathrm{Fe} / \mathrm{H}]$ & $\Delta T_{\text {eff }}$ \\
\hline$\Delta V$ & +0.01 & $-0.31 \pm 0.18$ & $-34 \pm 12$ \\
& -0.01 & $+0.41 \pm 0.28$ & $+31 \pm 12$ \\
$\Delta B$ & +0.01 & $+0.25 \pm 0.21$ & $-3 \pm 7$ \\
& -0.01 & $-0.17 \pm 0.12$ & $+0 \pm 6$ \\
$\Delta I$ & +0.01 & $+0.22 \pm 0.15$ & $+33 \pm 5$ \\
& -0.01 & $-0.18 \pm 0.12$ & $-34 \pm 6$ \\
$\Delta \log g$ & +0.10 & $-0.01 \pm 0.03$ & $+8 \pm 7$ \\
& -0.10 & $+0.01 \pm 0.04$ & $-8 \pm 8$ \\
\hline Random errors: & & & \\
Parameter & $\sigma$ & $\sigma[\mathrm{Fe} / \mathrm{H}]$ & $\sigma\left(T_{\text {eff }}\right)$ \\
\hline$\sigma(B-V)=\sigma(V-I)$ & 0.01 & 0.26 & 35 \\
$\sigma[E(B-V)]$ & 0.01 & 0.05 & 44 \\
\hline
\end{tabular}

Notes: The table shows the errors (computed minus model values) of the photometric $[\mathrm{Fe} / \mathrm{H}]$ and $T_{\text {eff }}$ for various assumed errors in the input parameters. All other input parameters are left at their theoretical values, except for the parameter indicated. The symbol $\Delta$ in the parameter column means 'shifted minus theoretical' values. We used Gaussian noise in the random simulations (last two rows). In computing the assemble values, we used $4 \sigma$ clipping. The errors depend on the actual parameter regime (see, e.g., Fig. 1). The errors shown in this table are average values including the full parameter range.

also in the size of scatter (due to the various values of $T_{\text {eff }}$ and $\log g$ ). On the other hand, it is conceivable that if we are interested in relative abundances, then the method is more accurate, especially at higher metallicities.

To get a closeup on the effects of other possible systematic shifts and random errors, we summarize the resulting $[\mathrm{Fe} / \mathrm{H}]$ errors in Table 1 (for completeness, we also show $\left.T_{\text {eff }}\right)$. As it is expected, photometric errors play the most important role, while errors due to reddening are smaller. Gravity estimates can be especially erroneous without affecting the derived metallicity.

\section{$3[\mathrm{Fe} / \mathrm{H}]$ distributions in the MCs}

We applied the above method of $[\mathrm{Fe} / \mathrm{H}]$ determination to the Cepheid database published by Udalski et al. (1999). We used their periods, intensity-averaged magnitudes and reddening values as given in their *.tab files 12 To avoid large errors due to observational noise, as mentioned above, we accepted solutions only if they satisfied the $\mathcal{D}<0.001$ criterion. In addition, we also employed a $3 \sigma$ clipping to filter out extreme values. The resulting $[\mathrm{Fe} / \mathrm{H}]$ distributions are shown in Fig. 2.

\footnotetext{
${ }^{1} \mathrm{ftp} / / /$ sirius.astrouw.edu.pl/ogle/ogle2/var_stars/lmc/cep/catalog

${ }^{2} \mathrm{ftp}$ ://sirius.astrouw.edu.pl/ogle/ogle2/var_stars/smc/cep/catalog/
} 

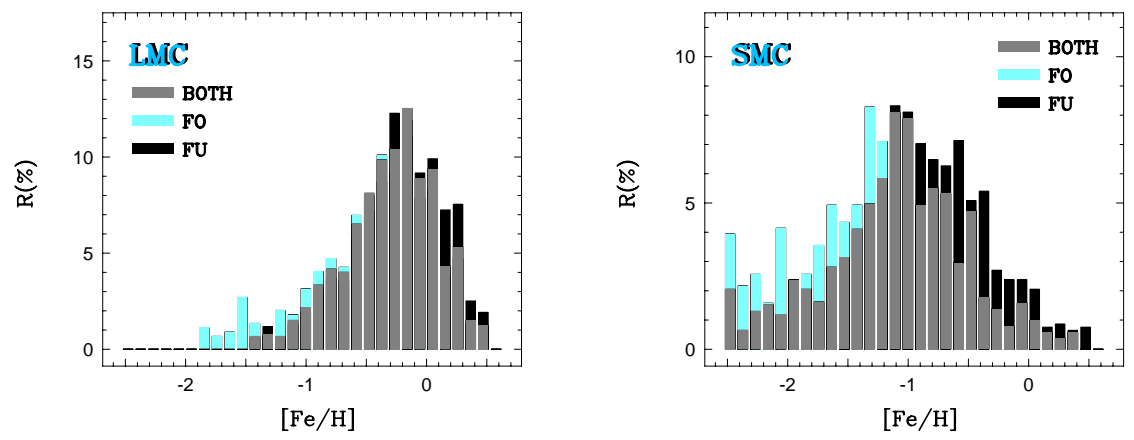

Fig. 2. Metallicity distributions computed directly from the observed $B V I$ colors.

Table 2. Average photometric metallicities for the Magellanic Cloud Cepheids

\begin{tabular}{cccccc}
\hline \hline Cloud & $N_{\mathrm{FU}}$ & {$[\mathrm{Fe} / \mathrm{H}]_{\mathrm{FU}}$} & $N_{\mathrm{FO}}$ & {$[\mathrm{Fe} / \mathrm{H}]_{\mathrm{FO}}$} & {$[\mathrm{Fe} / \mathrm{H}]_{\mathrm{FU}}-[\mathrm{Fe} / \mathrm{H}]_{\mathrm{FO}}$} \\
\hline LMC & 676 & $-0.27 \pm 0.02$ & 437 & $-0.43 \pm 0.02$ & $0.16 \pm 0.03$ \\
$\mathrm{SMC}$ & 941 & $-0.97 \pm 0.02$ & 519 & $-1.23 \pm 0.03$ & $0.26 \pm 0.03$ \\
\hline
\end{tabular}

It is clearly seen that there is a surplus of first overtone mode variables at the low-metallicity end of the distributions in both clouds. The same is true for the fundamental mode variables at the high-metallicity end. In both clouds the metallicity spread is large but it is clear that most of this comes from observational errors (e.g., the spread is larger in the SMC, because of the larger photometric errors). When computing the average metallicities, we get the result shown in Ta-

ble 2. Based on the formal errors of the mean values, the differences in the overall metallicities seem to be significant and are exhibited in the same sense in both clouds. We note that: (i) the average photometric metallicities are quite close to the 'canonical' values (e.g., Udalski et al. 1999); (ii) the derived lower metallicity for the SMC FO variables is the same as suggested by Cordier et al. (2003) for the low-luminosity Cepheids.

\section{Combination with the PLC relations?}

In the direct computation of $[\mathrm{Fe} / \mathrm{H}]$ from the observed color indices, large errors are introduced due to the high sensitivity to the observational errors. In principle, a considerable fraction of this error would be possible to eliminate if we could employ the period-luminosity-color (PLC) relations and use only the $I$-band magnitudes and the period to compute the color indices. Let us assume that for each reddeningfree magnitude we have the same type of relations for all Cepheids pulsating, e.g., in the fundamental mode:

$$
W_{\mathrm{B}-\mathrm{V}}=I-1.86(B-V)=a_{0}+a_{1} \log P_{0},
$$


Title : will be set by the publisher

$$
W_{\mathrm{V}-\mathrm{I}}=I-1.50(V-I)=b_{0}+b_{1} \log P_{0},
$$

where the $a_{i}$ and $b_{i}$ coefficients are independent of the metallicity. Similar relations are assumed to be hold also for the FO variables. The obvious advantage of the above relations is that the errors in the computed color indices become highly correlated through the errors of the directly used color $I$. Indeed, if we test this method on the stellar atmosphere models of Castelli et al. (1997), then, with an error of $\sigma_{I}=0.03$, we get $\sigma_{[\mathrm{Fe} / \mathrm{H}]}=0.06$ (we recall that independent errors of 0.01 in the color indices cause errors of $\sigma_{[\mathrm{Fe} / \mathrm{H}]}=0.26$ - see Table 1$)$. We note in passing that $T_{\text {eff }}$ will not become better estimated. With the above noise we get $\sigma_{T_{\text {eff }}}=65$. This high insensitivity of $[\mathrm{Fe} / \mathrm{H}]$ against observational noise can be understood by the inspection of the linear approximation of the colortemperature dependence in the above colors. The coefficients of the color terms are nearly cancelled out when solved for the metallicity with the aid of the above PLC relations (see Kovács \& Walker 1999).

Although the above method is very promising, there are considerable difficulties when looking at the assumptions in more detail. First of all, as it follows from the $M, L$ and $T_{\text {eff }}$ dependence of the pulsation period, the PLC relations should have some internal scatter, because the mass-luminosity relation is not a strict one. Therefore, the derived relation may depend on the stellar population studied and cannot be employed in a straightforward way to other systems. The population dependence is obvious in the case of PL relations and is widely discussed in the literature. Although PLC relations have been shown to be much less sensitive to such effects, in fine details we might face with similar problems as in the case of the PL relations. This question has recently been studied by Fiorentino et al. (2007). They showed that the PLCs based on the $B, V$ colors have significant systematic dependence on the metallicity. The effect is smaller but not negligible also in the near infrared colors. This has led the authors to estimate the metallicities of Galactic Cepheids which they showed to exhibit a fine correlation with the direct spectroscopic data of Andrievsky and coworkers.

There are also ambiguities in the derivation of the PLC relations. Udalski et al. (1999) employ a 2.5 $\sigma$-clipping and introduce various cuts in their MC sample. In this way they end up with the same slope in $W_{\mathrm{V}-\mathrm{I}}$ for the $\mathrm{FU}$ variables in both clouds. However, they note that the slopes for the FO variables might be different. Also, they did not study $W_{\mathrm{B}-\mathrm{V}}$, presumably due to the paucity of the $B$ data.

We derived the PLC relations in both unreddened colors as given by Eqs. (4.1) and (4.2). We do not apply any period constraint on the sample. For each class of variables the first 40 strongest outliers have been omitted iteratively. Table 3 gives the resulting regression parameters. By comparing the same types of variables in the two clouds, we see that there is a significant difference in $b_{1}$ for the FU variables. Although we use a slightly different expression for $W_{\mathrm{V}-\mathrm{I}}$ than Udalski et al. (1999), we think that this result is mainly due to the drop of the period constraint. We recall that Udalski et al. (1999) derived the same slope in both clouds within fairly narrow error limits. On the other hand, we agree with them on the $\mathrm{FO}$ variables: the $\mathrm{SMC}$ variables have a steeper slope in $W_{\mathrm{V}-\mathrm{I}}$ than the 
Géza Kovács: On the Cepheid Metallicity Dichotomy

Table 3. PLC relations for the Magellanic Clouds Cepheids

\begin{tabular}{cccccr}
\hline \hline & \multicolumn{2}{c}{$I-1.86(B-V)=a_{0}+a_{1} \log P$} \\
Cloud & Mode & $a_{0}$ & $a_{1}$ & $\sigma_{\text {fit }}$ & $N$ \\
\hline LMC & FU & $16.021 \pm 0.013$ & $-3.590 \pm 0.019$ & 0.109 & 732 \\
SMC & FU & $16.677 \pm 0.008$ & $-3.590 \pm 0.016$ & 0.166 & 1211 \\
LMC & FO & $15.444 \pm 0.008$ & $-3.495 \pm 0.023$ & 0.094 & 469 \\
SMC & FO & $16.150 \pm 0.007$ & $-3.604 \pm 0.028$ & 0.150 & 734 \\
\hline \multicolumn{5}{c}{$I-1.50(V-I)=b_{0}+b_{1} \log P$} \\
Cloud & Mode & $b_{0}$ & $b_{1}$ & $\sigma_{\text {fit }}$ & $N$ \\
\hline LMC & FU & $15.915 \pm 0.007$ & $-3.314 \pm 0.011$ & 0.064 & 732 \\
SMC & FU & $16.521 \pm 0.006$ & $-3.419 \pm 0.012$ & 0.132 & 1211 \\
LMC & FO & $15.405 \pm 0.006$ & $-3.397 \pm 0.015$ & 0.063 & 469 \\
SMC & FO & $16.011 \pm 0.006$ & $-3.556 \pm 0.025$ & 0.134 & 734 \\
\hline
\end{tabular}

ones in the LMC. A similar effect is seen for $W_{\mathrm{B}-\mathrm{V}}$. Accidentally, the FU variables behave in the same way in both clouds in respect of the above parameter. It is also important to note that the $W_{\mathrm{B}-\mathrm{V}}$ index yields $\sim 0.15 \mathrm{mag}$ larger value for the relative distance modulus of the clouds than the 'canonical' value of 0.51 mag derived from the $W_{\mathrm{V}-\mathrm{I}}$ index.

If we disregard all the above inconsistencies and use the PLC method to compute $[\mathrm{Fe} / \mathrm{H}]$, we get results confirming the metallicity difference between the $\mathrm{FO}$ and FU Cepheids in both clouds. For the LMC we get average metallicities of -0.22 and -0.36 for the FU and FO variables, respectively. For the SMC we get -1.24 and -1.63 , that are considerable lower than the ones obtained directly from the color indices (however, the relative difference between the FU and FO variables remains nearly the same). In both clouds (but especially in SMC) we get a considerable decrease in the total metallicity range.

\section{Conclusions}

To lend further support to the hypothesis of systematic metallicity spread among the Magellanic Cloud Cepheids, we attempted to derive photometric metallicities from the OGLE BVI photometry. Aided by the stellar atmosphere models of Castelli et al. (1997) we have shown that first overtone variables have systematically lower abundances than fundamental ones. The effect is larger in the SMC, amounting to $\sim 0.3 \mathrm{dex}$ (vs. $\sim 0.2 \mathrm{dex}$ in the LMC). This result is in agreement with the ones suggested by the evolutionary models of Cordier et al. (2003) on the condition of instability strip crossing of low-luminosity Cepheids in the SMC. Also, the discrepancy between the pulsational and evolutionary ML relations (Beaulieu et al. 2001) can be largely cured by assuming lower metallicities. Yet another, independent result in fitting the periods of the $\mathrm{SO} / \mathrm{FO}$ Cepheids in the Magellanic Clouds shows that they require lower metallicities than their FO/FU counterparts (Kovács G. 2006). We think that all these results point toward the reality of the 
Title : will be set by the publisher

metallicity dichotomy between the FO and FU variables. Obviously, a massive spectroscopic survey of the Magellanic Clouds Cepheids (similar to that of Mottini et al. 2006) would be of great value, because the photometric abundances are of low accuracy and cannot tell the degree of the dichotomy and its more precise relation to the luminosity. We also attempted to use the PLC relations to derive more accurate color indices and thereby more precise abundances. Unfortunately this method remains somewhat doubtful due to controversies in the observed relations and due to inherent scatter of the theoretical ones (Fiorentino et al. 2007).

\section{Acknowledgments}

I would like thank to Robert for the exciting and cheerful years I was able to spend at the Physics Department of the University of Florida. This long-term collaboration was very pleasant and fruitful for me. I feel privileged to be a member of his long list of postdoctoral fellows. Thanks are due to István Dékány for the careful reading of the manuscript. This work has been supported by the Hungarian Scientific Research Foundation (OTKA) grant K-60750.

\section{References}

Beaulieu, J. P., Buchler, J. R. \& Kolláth, Z., 2001, A\&A, 373, 164

Bono, G., Caputo, F. \& Stellingwerf, R. F., 1995, ApJS, 99, 263

Castelli F., Gratton R. G. \& Kurucz R. L., 1997, A\&A 318, 841

Clementini, G. et al., 1995, AJ 110, 2319

Cordier, D., Goupil, M. J. \& Lebreton, Y., 2003, A\&A, 409, 491

Dékány, I., Kovács, G., Jurcsik, J. et al., 2008, MNRAS, submitted

Fiorentino, G, Marconi, M., Musella, I. \& Caputo, F., 2007, A\&A, 476, 863

Rogers, F. J. \& Iglesias, C. A., 1992, ApJS, 79, 507

Kovács G., 2000, A\&A, 360, L1

Kovács G., 2006, MmSAI, 77, 160

Kovács G. \& Walker A. R., 1999, ApJ, 512, 271

Luck, R. E., Kovtyukh, V. V., \& Andrievsky, S. M., 2006, AJ, 132, 902

Moskalik, P. \& Dziembowski, W. A., 1992, A\&A, 256, L5

Moskalik, P., Buchler, J. R. \& Marom, A., 1992, ApJ, 385, 685

Mottini, M., Romaniello, M., Primas, F. et al., 2006, MmSAI, 77, 156

Simon, N. R., 1982, ApJ, 260, L87

Taylor, B. J., 2003, A\&A, 398, 731

Udalski, A. et al., 1999, Acta Astron. 49, 201

Yong, D. \& Grundahl, F., 2008, ApJ, 672, L29 